\title{
A rheumatology based ultrasound service in clinical practice - results from a busy urban early inflammatory arthritis service
}

\author{
Authors: Maathu Ratnaraj, ${ }^{\mathrm{A}}$ Tina Parish ${ }^{\mathrm{A}}$ and Tazeen Ahmed $^{\mathrm{B}}$
}

\section{Introduction}

The use of ultrasound (US) scans within a rheumatological setting has been increasing significantly over the past 10 years due to its ease of use and access compared to magnetic resonance imaging. Most published studies are set in a research setting with a structured emphasis on clinical diagnoses and outcomes. Here we look at real world data from an established rheumatology based US service which supports an early inflammatory arthritis service. The aim of this study was to look at the utilisation and outcomes of the rheumatology based US service in a busy urban general rheumatology department.

\section{Materials and methods}

The study sample included 141 patients who were referred for a US scan from March to August 2018; data was collected from electronic patient records (Cerner). Data was analysed using MS Excel 2010

\section{Results and discussion}

$76.6 \%$ of patients were referred for a US scan for a synovitis screen. $54 \%$ of scans were conducted on the hands and wrist of patients. $6.4 \%$ of these patients were anti-cyclic citrullinated peptide positive and $22 \%$ were rheumatoid factor positive. $18 \%$ of scans led to a diagnosis of inflammatory arthritis, $9 \%$ psoriatic arthritis and 3\% rheumatoid arthritis. 35\% were diagnosed with osteoarthritis. $23 \%$ had further investigations recommended after the scan. Most US scans led to a therapeutic outcome, see Table 1.

\section{Conclusion}

The majority of scans supported a diagnosis of inflammatory arthritis and a fifth required further investigations. This study shows that US is an important tool in the assessment of inflammatory

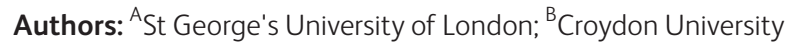
Hospital
Table 1. Outcomes from the rheumatology

ultrasound scan appointment

\begin{tabular}{ll} 
Outcome of scan & Percentage of patients (\%) \\
Start DMARD & 26 \\
Same DMARD & 2 \\
Stop DMARD & 1 \\
Referral to physiotherapy & 14 \\
Referral to hand/feet therapy & 7 \\
Others & 13 \\
Did not attend & 3 \\
None of the above & 34 \\
\hline DMARD = disease-modifying anti-rheumatic drug.
\end{tabular}

arthritis but is often not sufficient in itself to provide a complete diagnosis. However, the US service does provide useful outcomes where there is diagnostic dilemma. 